\title{
Epidemiological and molecular features of norovirus infections in Italian children affected with acute gastroenteritis
}

\author{
M. C. MEDICI ${ }^{1}$, F. TUMMOLO ${ }^{1}$, V. MARTELLA ${ }^{2}$, C. $_{\text {CHEZZI }}{ }^{1}$, \\ M. C. ARCANGELETTI ${ }^{1}$, F. DE CONTO $^{1}$ AND A. CALDERARO ${ }^{1}$ \\ ${ }^{1}$ Unit of Microbiology and Virology, Department of Clinical and Experimental Medicine, University of Parma, \\ Parma, Italy \\ ${ }^{2}$ Department of Veterinary Medicine, University Aldo Moro of Bari, Valenzano, Bari, Italy
}

Received 4 April 2013; Final revision 18 October 2013; Accepted 11 December 2013; first published online 20 January 2014

\section{SUMMARY}

During a 5-year (2007-2011) surveillance period a total of $435(15 \cdot 34 \%)$ of 2834 stool specimens from children aged $<14$ years with acute gastroenteritis tested positive for norovirus and 217 strains were characterized upon partial sequence analysis of the polymerase gene as either genogroup (G)I or GII. Of the noroviruses, $99 \cdot 2 \%$ were GII with the GII.P4 genotype being predominant (80\%). GII.P4 variants (Yerseke 2006a, Den Haag 2006b, Apeldoorn 2008, New Orleans 2009) emerged sequentially during the study period. Sequence analysis of the capsid gene of 57 noroviruses revealed that $7 \cdot 8 \%$ were recombinant (ORF1/ORF2) viruses including GII.P7_GII.6, GII.P16_GII.3, GII.P16_GII.13, GII.Pe_GII.2, and GII.Pe_GII.4, never identified before in Italy. GII.P1_GII.1, GII.P2_GII.1, GII.P3_GII.3 and GII.P6_GII.6 strains were also detected. Starting in 2011 a novel GII.4 norovirus with 3-4\% nucleotide difference in the polymerase and capsid genes from variant GII.4 New Orleans 2009 was monitored in the local population. Since the epidemiology of norovirus changes rapidly, continuous surveillance is necessary to promptly identify the onset of novel types/variants.

Key words: Gastroenteritis, GII.4 variant, norovirus, recombination.

\section{INTRODUCTION}

Noroviruses (NoVs) represent one of the most common causes of enteric disease in humans [1, 2]. The prevalence of NoV in children with acute gastroenteritis ranges from $6 \%$ to $48 \%$ [1-3]. NoVs cause significant mortality and morbidity worldwide with 200000 deaths each year in children aged $<5$ years, 64000 paediatric hospital admissions and 900000 paediatric hospital visits in developed countries each year [4].

\footnotetext{
* Author for correspondence: Professor M. C. Medici, Unit of Microbiology and Virology, Department of Clinical and Experimental Medicine, University of Parma, Viale Antonio Gramsci, 14-43126 Parma, Italy.

(Email: mariacristina.medici@unipr.it)
}

NoVs, family Caliciviridae, are non-enveloped viruses with a single-stranded, positive-sense, polyadenylated RNA genome of about 7500 nucleotides in length [5], organized into three open reading frames (ORFs), ORF1, 2, and 3, which encode a polyprotein including the RNA-dependent RNA polymerase (RdRp), the major capsid protein, and a small capsid protein, respectively.

NoVs encompass five distinct genogroups (GI-GV) with GI, GII and GIV infecting humans. The NoV genogroups are further divided into genotypes and variants based on sequence diversity [5]. Along with point mutations, recombination events seem to contribute largely to the genetic variability of NoVs [6]. The ORF1/ORF2 junction region is a recombination 
hotspot and this creates various combinations of RdRp and capsid types [6]. Multi-target characterization of NoVs in the RdRp region (diagnostic regions $\mathrm{A}$ and $\mathrm{B}$ ) [7-10] and capsid gene (diagnostic regions C-D) $[11-14]$ is therefore necessary to characterize precisely NoVs.

Thus far, 14 GI and 29 GII RdRp genotypes, and eight GI and 23 GII capsid genotypes have been described. RdRp genotypes are designated by a capital $\mathrm{P}$ (for 'polymerase'), followed by an arabic number, i.e. GII.P4, in order to avoid confusion with capsid type nomenclature [15].

Over the last decade the most common combinations of $\mathrm{RpRd} /$ capsid genotypes in the circulating NoV strains were GII.P4_GII.4 and GII.Pb_GII.3 [16]. GII.4 NoVs are highly heterogeneous genetically and evolve quickly, generating new variants every 2 or 3 years [17-20]. Some GII.4 variants (Farmington Hills 2002, Hunter 2004, Yerseke 2006a, Den Haag 2006b, Apeldoorn 2008, New Orleans 2009, Sydney 2012) in the last decade have reached a global distribution $[16,21]$.

While reports on NoV infections/outbreaks are numerous, studies describing the molecular epidemiology of NoVs associated with sporadic gastroenteritis are scanty, usually spanning a short time frame [16]. However, gathering information on the epidemiology of NoVs is pivotal for tracking the origin of infections, understanding the dynamics of NoV circulation and devising appropriate public health intervention strategies. Patients hospitalized with gastroenteritis are a good target for gathering epidemiological information on enteric pathogens circulating in the community.

This study describes the results of a 5-year (2007-2011) hospital-based surveillance activity for sporadic NoV infections associated with gastroenteritis in the area of Parma, Northern Italy. Molecular characterization in the ORF1 and ORF2 of a representative selection of $\mathrm{NoV}$ strains was achieved in order to generate a reliable picture of NoV molecular epidemiology.

\section{MATERIALS AND METHODS}

\section{Stool specimens}

Stool samples of 2834 children (age range 0-169 months, median age 33 months) with acute gastroenteritis attending the Maternal Infantile Department of the University Hospital of Parma, Northern Italy, as inpatients $(84 \cdot 7 \%)$ and outpatients $(15 \cdot 3 \%)$ during
January 2007-December 2011 were submitted for virological investigations at the Virological Unit of the Department of Pathology and Laboratory Medicine of the same University Hospital.

The faecal specimens were clarified in $10 \%(\mathrm{w} / \mathrm{w})$ suspensions in phosphate-buffered saline (PBS, pH 7.2) and analysed by electron microscopy (EM), latex agglutination for group A rotavirus (RV) and adenovirus (AdV) (Orion Diagnostica, Finland), and inoculated onto cell cultures as previously reported [22]. Cytopathogenic agents were identified by EM, neutralization tests using Lim Benyesh-Melnick antisera pools [23, 24] or EM and polyacrylamide gel electrophoresis [25, 26].

\section{RNA extraction and detection of $\mathrm{NoV}$ in clinical samples}

For NoV detection, nucleic acids were automatically extracted by the NucliSens easyMAG instrument (bioMérieux, France) from $500 \mu \mathrm{l}$ of stool suspension in a final volume of $25 \mu \mathrm{l}$. Following the manufacturer's instructions, detection of NoV RNA was performed using the RT Kit Plus (Nanogen Advanced Diagnostics, Italy) for reverse transcription (RT), and the NoV Oligomix Alert kit (Nanogen Advanced Diagnostics) for nested PCR (nPCR), targeting a 174 176 bp fragment of NoV RdRp, using CPE-RNA (Nanogen Advanced Diagnostics) as an internal RNA standard control.

Stool samples, faecal suspensions and first-round PCR products were stored at $-20^{\circ} \mathrm{C}$ until use.

\section{Nucleotide sequencing and molecular typing}

Sequence analysis was undertaken on amplicons (327 bp in length), yielded with primer pair JV12/ JV13 that target the ORF1 RdRp region A [22], from a set of NoV-positive specimens, selected according to the seasonal distribution of $\mathrm{NoV}$ infections.

For potential recombinant genomes of NoVs, and to exclude cases of mixed infection, the partial ORF1 RdRp and ORF2 capsid gene sequences along with the junction between RdRp and capsid (region $\mathrm{A}-\mathrm{C}, 1110 \mathrm{bp}$ ) of a selection of strains representative of the $\mathrm{RdRp}$ lineages and sublineages were amplified by RT-PCR using primers JV12 and G2R1 [8, 14].

The Superscript One-Step RT-PCR for Long Templates kit (Invitrogen, USA) was used. Briefly, $5 \mu \mathrm{l}$ extracted RNA was added to $12.5 \mu 1$ of $2 \times$ reaction 
mix, $4 \cdot 5 \mu \mathrm{l} \mathrm{H}_{2} \mathrm{O}, 0 \cdot 5 \mu \mathrm{l}$ R/Platinum Taq HiFi mix, containing $10 \mathrm{pmol}$ of each primer. The reaction was performed in a PerkinElmer 9700 Thermal Cycler (Applied Biosystems, USA) with the following temperature conditions: $42{ }^{\circ} \mathrm{C}$ for $60 \mathrm{~min}, 94^{\circ} \mathrm{C}$ for $2 \mathrm{~min}, 40$ cycles at $94^{\circ} \mathrm{C}$ for $1 \mathrm{~min}, 50^{\circ} \mathrm{C}$ for $2 \mathrm{~min}$ and $68^{\circ} \mathrm{C}$ for $2 \mathrm{~min}$, followed by $72^{\circ} \mathrm{C}$ for $10 \mathrm{~min}$. Finally, amplicons of ORF2 region D (254 bp) were also produced from GII.4 strains using published primers and protocols [11].

The PCR products were electrophoresed on ethidium bromide-stained $2 \%$ agarose gel at $120 \mathrm{~V}$ for $40 \mathrm{~min}$, visualized under UV light by GelDoc XR instrument (Bio-Rad Laboratories, USA), and purified using QIAquick Gel Extraction kit (Qiagen, Germany). Sequencing was performed using the PCR primers and BigDye Terminator Cycle Sequencing kit (Applied Biosystems) on an automated DNA sequencer (ABI 3730, PerkinElmer-Applied Biosystems, USA). All sequences were typed with the genotyping tool for NoVs (http://www.rivm.nl/ $\mathrm{mpf} /$ norovirus/typingtool) [27].

\section{Phylogenetic analysis of NoV GII.4}

In order to investigate the genetic relationship among GII.4 strains, phylogenetic analysis was performed with the generated RdRp and capsid sequences for NoV GII.4 strains identified during the study period. The sequences were analysed using MEGA 5.0 software [28]. Maximum composite likelihood was used as the substitution method, and the neighbour-joining method was used for construction of the phylogenetic tree. The reliability of the phylogenetic tree was assessed by bootstrap re-sampling over 1000 replicates. The BLAST web-based analysis tool with default values was used to find homologous hits in the sequence database (GenBank, NCBI). The nucleotide sequences have been deposited in GenBank under accession numbers KF743088-KF743123 (RdRp region), and KF743064-KF743087 (capsid region).

\section{Statistical analysis}

Statistical analysis was performed in order to compare the rates of detection of NoVs in different age groups and seasons, and to understand whether some NoV genotypes may preferentially infect some age groups, or whether patients with co-infections are more susceptible to some NoV strains. The $\chi^{2}$ test was applied to the data, using GraphPad instat v. 3.05 for
Windows (GraphPad Software, USA), with $P<0.05$ considered to be significant.

\section{RESULTS}

\section{Epidemiological features and NoV genotyping}

NoV was detected as a single pathogen in 368 (12.98\%, with a range from $9 \cdot 8 \%$ in 2011 to $17 \cdot 2 \%$ in 2008$)$ cases whereas, $50(1 \cdot 8 \%), 13(0 \cdot 2 \%)$ and four $(0 \cdot 1 \%)$ samples had mixed infections with $\mathrm{RV}$, AdV and enterovirus, respectively (Table 1$)$. A total of $296(68.04 \%)$ of the NoV-positive samples were from children aged $<3$ years $(P=0 \cdot 0001)$ (Fig. 1). Of this group of patients, $46(15 \cdot 54 \%)$ children showed mixed infection with RV in 35 cases (52.23\%), AdV in nine cases $(13.43 \%)$ and enterovirus in two cases $(2 \cdot 98 \%)$. The monthly distribution plot showed that $\mathrm{NoV}$ was detected throughout the year, except in July 2007 and July-August 2011, with an increasing number of infections during the autumn-winter per$\operatorname{iod}(73 \cdot 56 \% ; 320 / 435)(P=0 \cdot 0001)$ (Fig. 2).

A total of $217(49 \cdot 88 \%)$ NoV strains were sequenced in the RdRp (region A). Seven typed NoVs (3.22\%) belonged to GI, including four different genotypes (GI.P1, GI.P2, GI.P4, GI.P8), and 210 (96.77\%) belonged to GII, of which $168(80 \%)$ were characterized as GII.P4 (Table 2, Fig. 3). Within the GII.P4 genotype, four distinct variants (Yerseke 2006a, Den Haag 2006b, Apeldoorn 2008, New Orleans 2009), were identified. The remaining $42(20 \%)$ GII NoVs included 19 (45\%) GII.Pb, eight (19\%) GII.P1, five (12\%) GII.Pg, two (4.8\%) GII.P2, two (4.8\%) GII. P7, two (4.8\%) GII.P16, two (4.8\%) GII.Pe, one $(2 \cdot 4 \%)$ GII.P3, and one $(2 \cdot 4 \%)$ GII.P6.

Sequencing of the A-C genomic fragment of 57 strains allowed the identification of 17 potential NoV recombinants with a total of 12 different combinations of RdRp and capsid genotypes: GI.P1_GI.3, GII.P7_GII.6, GII.P16_GII.3, GII.P16_GII.13, GII.Pb_GII.2, GII.Pb_GII.3, GII.Pb_GII.13, GII. Pb_GII.21, GII.Pg_GII.1, GII.Pg_GII.12, GII. Pe_GII.2, and GII.Pe_GII.4 2007. For the other 40 strains, the capsid genotype matched the RdRp-based identification (Table 2). Multiple genotypes were found in co-infection with other enteric viral pathogens. Out of 19 genotypes identified in 67 mixed infections, eight were GII.P4 Den Haag 2006b, five were GII.P4 New Orleans 2009, two were GII.P4 Yerseke 2006a, and one each for GII.P4 Apeldoorn 2008, GII.Pb, GII.P1, and GII.P2. No statistical correlation 


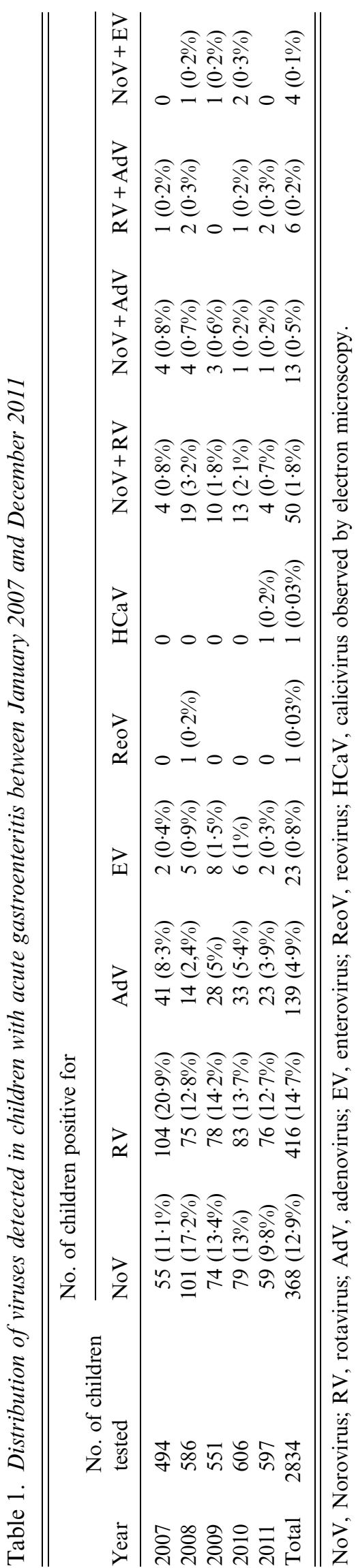

was found between genotypes and co-infections. Similarly, different NoV genotypes were found in various age groups. The distribution in age groups of the NoV genotypes matched the distribution of NoV prevalences by age. A statistical correlation was observed between GII.P4 Den Haag 2006b NoV and age $<3$ years $(P=0 \cdot 0078)$.

\section{Phylogenetic analysis of GII.4}

Phylogenetic clustering with GII.4 reference strain sequences from Noronet European genotyping tool displayed the genetic relationships among the 168 RdRp GII.4 genotypes detected in this study. In order to eliminate redundancy and prepare a readable figure, 36 sequences representative of the topology of branching of the RdRp GII.4 phylogenetic tree were used to prepare the tree in Figure $4 a$.

The NoV GII.P4 strains grouped in two clades with different lineages and sublineages in the phylogenetic tree. One clade including the Italian NoVs belonged to GII.P4 Yerseke 2006a, Apeldoorn 2008 and New Orleans 2009 (bootstrap values 50-79\%) and the second clade included the Italian GII.P4 Den Haag 2006b strains (bootstrap value 79\%). The branching order showed that almost all the GII.P4 New Orleans 2009 strains detected in 2011 formed a close divergent lineage (bootstrap value 53\%), that have a $4 \%$ nucleotide difference from the GII.P4 New Orleans 2009 strains. The 2011 GII.P4 strains were genetically highly homogenous $(99 \%$ nucleotide identity to each other in the $1100 \mathrm{bp} \mathrm{A}-\mathrm{C}$ genomic fragment).

The capsid phylogenetic tree of $24 \mathrm{NoV}$ Italian GII.4 sequences identified in this study showed that the clustering patterns were consistent with those observed in the RdRp-based analysis with the divergent lineage of the GII.4 New Orleans 2009 strains detected in 2011 (bootstrap value 45\%) (Fig. 4b). This lineage was phylogenetically distinct also from the new GII.4 Sydney 2012 reference strain. In both the capsid regions $\mathrm{C}$ and $\mathrm{D}$, the 2011 GII.4 viruses differed by 3-4\% from the GII.4 New Orleans 2009 variant.

\section{DISCUSSION}

$\mathrm{NoV}$ is regarded as a major aetiological agent of sporadic diarrhoea in infants and young children [1-5]. During 5 years of surveillance for viral agents in sporadic cases of childhood acute gastroenteritis in Parma, Northern Italy, NoV was detected in $15 \cdot 34 \%$ of children. The prevalence rates of NoV infection (with 


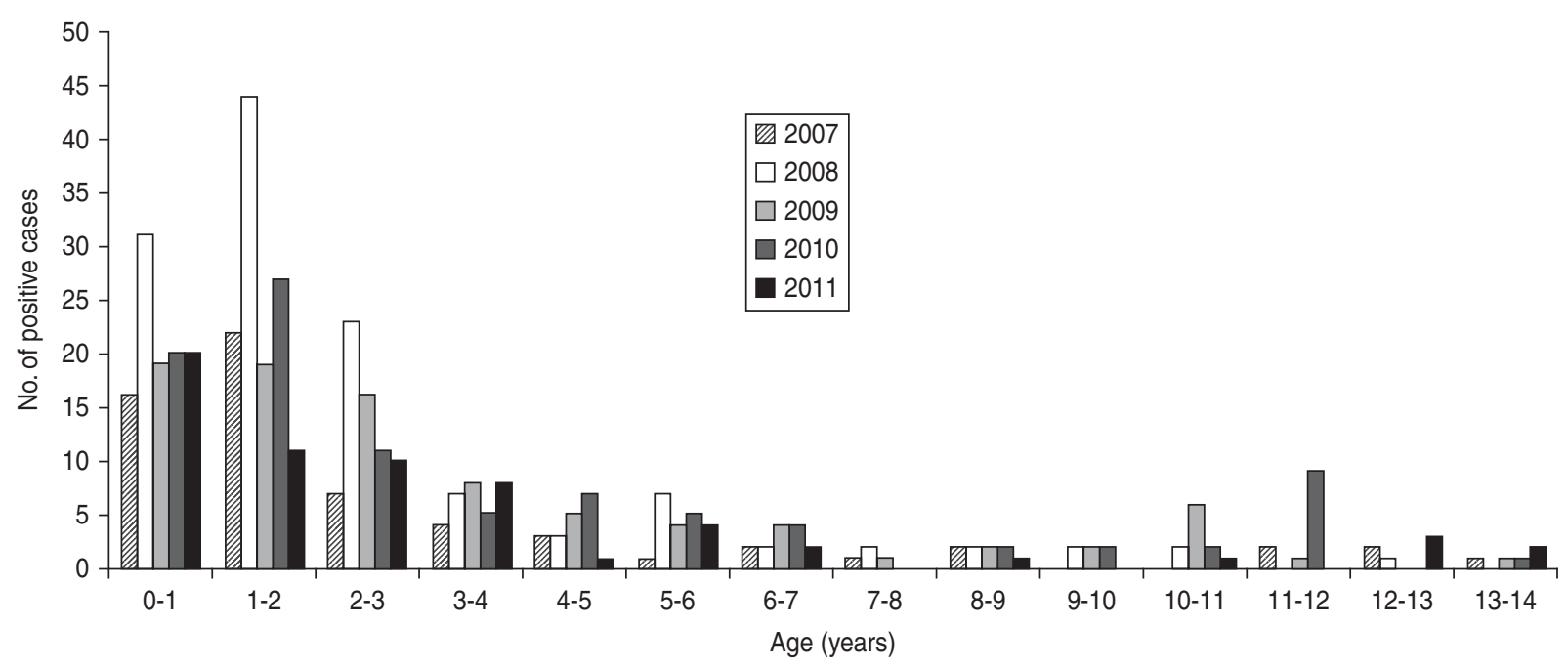

Fig. 1. Age distribution of children with norovirus-associated gastroenteritis (January 2007-December 2011).

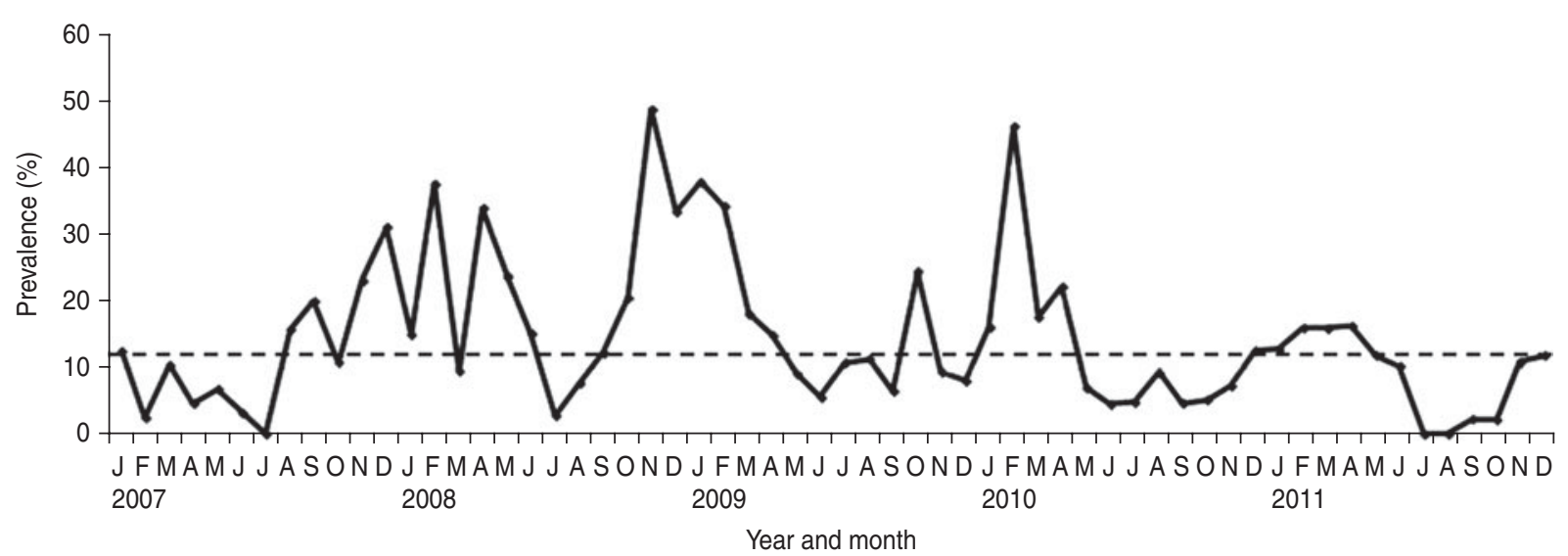

Fig. 2. Monthly distribution of sporadic cases of norovirus-associated gastroenteritis (January 2007-December 2011). The horizontal dotted line on the graph represents the median percentage of positive cases per month.

NoV as single pathogen) ranged between $9 \cdot 8 \%$ in 2011 and $17 \cdot 2 \%$ in 2008 , revealing annual variations, as observed elsewhere [16]. NoV infection occurred at higher frequency in children aged $<3$ years $(68.04 \%$ in this study), an age-related pattern that has been reported in other studies, confirming the relevance of NoV as a cause of severe gastroenteritis requiring hospitalization in young children [29-40]. In temperate climates NoV infections usually show a seasonal pattern with a peak during the cold months [41-43]. In this study, seasonal peaks of NoV activity were revealed between January and February every year from 2007 to 2010, while in 2011 the activity was prolonged and a peak was reached in April. Moreover, a summer peak was observed in August in 2009. The peaks of NoV activity tended to overlap with or to anticipate the peaks of RV activity in all the surveyed years, except in 2011, when this pattern was inverted (data not shown).

Genotyping of the NoV strains identified during 2007-2011 revealed that different NoV genotypes did co-circulate during the study period and that several reported GII.4 variants were also detected in this cohort. The predominance of GII.4 NoVs $(77 \cdot 4 \%)$ is consistent with the literature $[8,16,17,20]$. NoV GII.4 strains appear to evolve with genetic drift and shift mechanisms, with the periodic onset and rapid global spread of pandemic variants [8, 16, 17, 20]. This temporal pattern of evolution was also documented in our study. Variant GII.4 Yerseke 2006a was detected at a high frequency during 2007 and variant GII.4 Den Haag 2006b was predominant during 2007-2009, while variant GII.4 Apeldoorn 2008 was detected sporadically during 2009-2010. In 2010, 
Table 2. Number of norovirus genotypes detected in Parma, Italy, from 2007 to 2011

\begin{tabular}{|c|c|c|c|c|c|c|c|c|c|c|c|c|}
\hline \multirow{2}{*}{$\begin{array}{l}\text { Genotype of norovirus } \\
\text { No. norovirus detected }\end{array}$} & \multicolumn{2}{|l|}{2007} & \multicolumn{2}{|l|}{2008} & \multicolumn{2}{|l|}{2009} & \multicolumn{2}{|l|}{2010} & \multicolumn{2}{|l|}{2011} & \multicolumn{2}{|l|}{ Total } \\
\hline & 63 & & 125 & & 88 & & 95 & & 64 & & 435 & \\
\hline Region/s sequenced ( $n$ ) & $\mathrm{A}(50)$ & $\begin{array}{l}A+B+C \\
(16)\end{array}$ & A (57) & $\begin{array}{l}A+B+C \\
(6)\end{array}$ & A (42) & $\begin{array}{l}A+B+C \\
(12)\end{array}$ & A (41) & $\begin{array}{l}A+B+C \\
(12)\end{array}$ & A (27) & $\begin{array}{l}A+B+C \\
(10)\end{array}$ & A (217) & $\begin{array}{l}A+B+C \\
(57)\end{array}$ \\
\hline \multicolumn{13}{|l|}{ GI } \\
\hline GI.P1_GI.3 & I & I & I & I & 1 & 1 & l & l & I & l & 1 & 1 \\
\hline GI.P2_GI.2 & I & l & 1 & 1 & / & / & l & l & l & l & 1 & 1 \\
\hline GI.P4_GI.4 & I & I & I & I & 1 & 1 & I & I & 3 & 1 & 4 & 2 \\
\hline GI.P8_GI.8 & I & l & 1 & 1 & I & l & l & l & / & / & 1 & 1 \\
\hline \multicolumn{13}{|l|}{ GII } \\
\hline GII.P1_GII.1 & 1 & I & 4 & l & 1 & I & l & l & 2 & 1 & 8 & 1 \\
\hline GII.P2_GII.2 & / & l & 1 & l & 1 & 1 & l & l & l & / & 2 & 1 \\
\hline GII.P3_GII.3 & 1 & 1 & l & l & / & / & l & l & l & l & 1 & 1 \\
\hline $\begin{array}{l}\text { GII.P4 Yerseke 2006a_GII.4 } \\
\text { Yerseke 2006a* }\end{array}$ & 11 & 3 & 3 & I & 2 & 2 & l & l & I & l & 16 & 5 \\
\hline $\begin{array}{l}\text { GII.P4 Den Haag 2006b_GII.4 } \\
\text { Den Haag 2006b* }\end{array}$ & 28 & 7 & 45 & 4 & 21 & I & 8 & 2 & 1 & l & 103 & 13 \\
\hline $\begin{array}{l}\text { GII.P4 Apeldoorn 2008_GII.4 } \\
\text { Apeldoorn } 2008\end{array}$ & I & I & I & l & 4 & 2 & 1 & I & I & l & 5 & 2 \\
\hline $\begin{array}{l}\text { GII.P4 New Orleans 2009_GII.4 } \\
\text { New Orleans } 2009\end{array}$ & I & I & I & I & I & I & 26 & 6 & 1 & l & 27 & 6 \\
\hline $\begin{array}{l}\text { GII.P4 New Orleans 2009_ GII.4 } \\
\text { New Orleans 2009* } \\
\quad \text { (novel strain) }\end{array}$ & I & I & l & I & l & l & l & I & 17 & 5 & 17 & 5 \\
\hline GII.P6_GII.6 & I & l & l & l & 1 & 1 & l & l & l & I & 1 & 1 \\
\hline GII.P7 & l & I & l & l & 1 & I & l & l & l & l & 1 & / \\
\hline GII.P7_GII.6 & I & I & l & l & / & I & l & l & 1 & 1 & 1 & 1 \\
\hline GII.P16_GII.3 & I & I & I & I & I & I & l & I & 1 & 1 & 1 & 1 \\
\hline GII.P16_GII.13 & / & I & / & l & I & l & 1 & 1 & / & / & 1 & 1 \\
\hline GII.Pb & 4 & I & 2 & I & 5 & l & l & l & l & I & 11 & 1 \\
\hline GII.Pb_GII.2 & 1 & 1 & l & 1 & l & I & l & I & I & I & 1 & 1 \\
\hline GII.Pb_GII.3 & 3 & 3 & I & I & 1 & 1 & I & I & I & l & 4 & 4 \\
\hline GII.Pb_GII.13 & 1 & I & I & I & 2 & 2 & l & I & I & l & 2 & 2 \\
\hline GII.Pb_GII.21 & 1 & 1 & I & l & l & 1 & I & I & I & I & 1 & 1 \\
\hline GII.Pe_GII.2 & 1 & / & l & l & I & I & l & l & 1 & 1 & 1 & 1 \\
\hline GII.Pe_GII.4 2007 & I & I & I & l & 1 & 1 & l & I & l & I & 1 & 1 \\
\hline GII.Pg & I & I & I & I & l & l & 2 & I & I & l & 2 & I \\
\hline GII.Pg_GII.1 & I & I & I & I & I & I & 2 & 2 & I & I & 2 & 2 \\
\hline GII.Pg_GII.12 & I & I & I & I & I & I & 1 & 1 & I & I & 1 & 1 \\
\hline
\end{tabular}

* Four GII.P4 Yerseke 2006a_GII.4 Yerseke 2006a, 12 GII.P4 Den Haag 2006b_GII.4 Den Haag 2006b, three GII.P4 New Orleans 2009 GII.4 New Orleans 2009 and two GII.P4 New Orleans 2009_ GII.4 New Orleans 2009 (novel strain) strains were also sequenced in the capsid region D. 


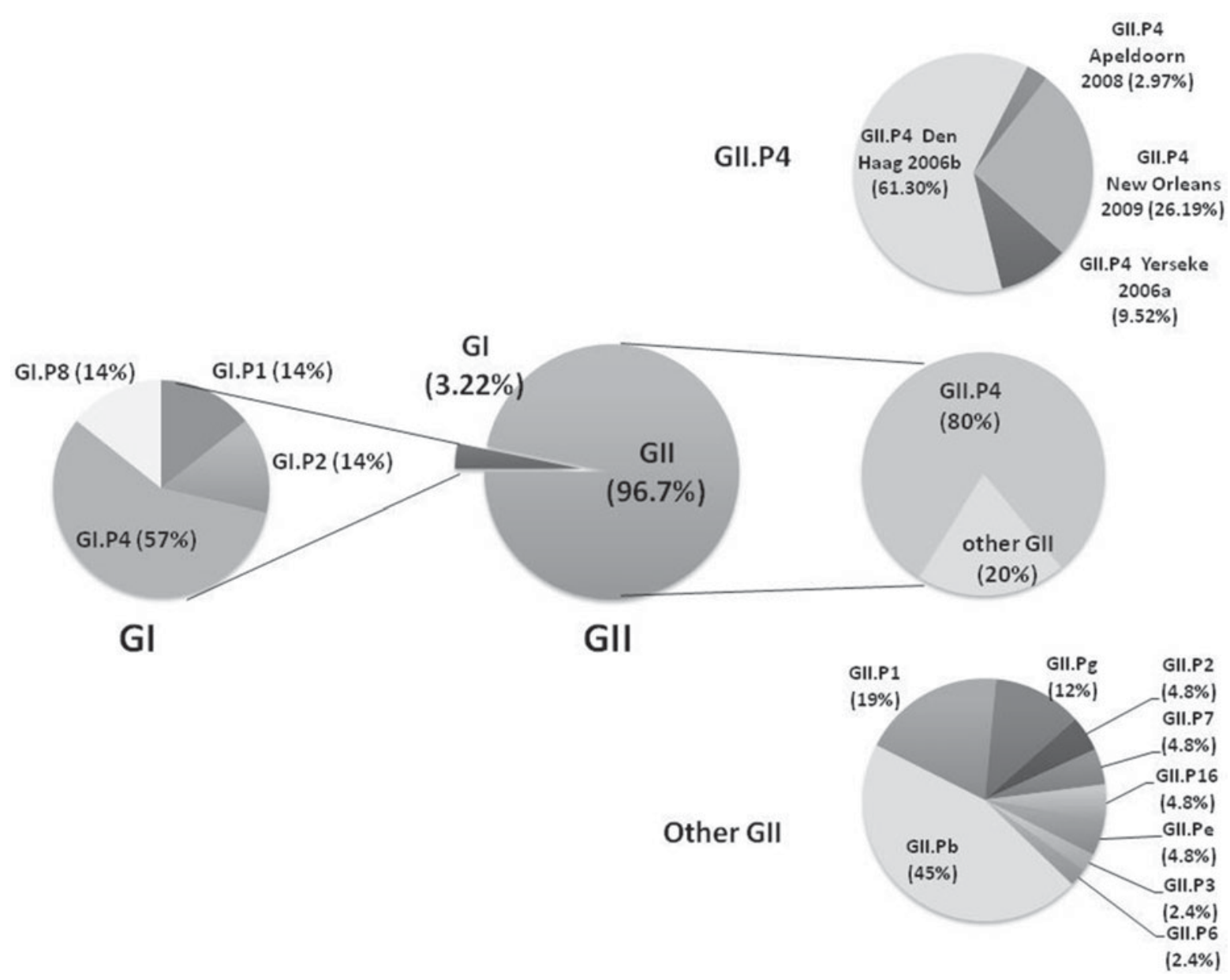

Fig. 3. Norovirus polymerase genotypes revealed from 217 children with gastroenteritis (January 2007-December 2011).

variant GII.4 New Orleans 2009 emerged and quickly replaced, almost completely, the former variants. Strains belonging to the GII.4 New Orleans 2009 variant clustered along with strains of GII.4 Yerseke $2006 \mathrm{a}$ in the same lineage in the phylogenetic tree showing their close evolutionary relationships. The evolutionary trend analysis confirmed the continuous pattern of co-divergence of GII.4 variants, with GII.4 Den Haag 2006b linked closely to GII.4 Farmington Hills 2002, and the GII.4 variants US 95_96 and Yerseke 2006a related to the variants Apeldoorn 2008 and Hunter 2004. The variant New Orleans 2009 derived from the pandemic variant Yerseke 2006a and the new GII.4 variant Sydney 2012 share a common ancestor in the capsid gene with GII.4 variants Apeldoorn 2008 and New Orleans 2009. Interestingly in this study, a novel GII.4 strain, descending from GII.4 variant New Orleans 2009, was identified in 2011, showing that this variant is undergoing further diversification. This novel GII.4 strain differed by $4 \%$ nucleotides in the $\mathrm{RdRp}$ and by $3-4 \%$ nucleotides in both the $\mathrm{C}$ and $\mathrm{D}$ capsid regions from NoVs of GII.4 variant New Orleans 2009. The onset of this novel GII.4 strain was not associated with significant increased NoV activity, as the prevalence rates did not exceed the prevalence rates observed in the epidemic seasons 2007-2008, 2008-2009, and 20092010 (data not shown). This is not surprising as the onset and spread of novel GII.4 variants is not always related to increase in NoV activity [17]. Whether this novel GII.4 strain may represent a novel GII.4 variant should be assessed in larger and concerted epidemiological studies and with analysis of the data retrospectively. However, hospital-based surveillance studies can offer a reliable approach to monitor promptly the onset on novel NoV strains and variants in a settled population.

NoV RdRp genotypes GII.P16, GII.Pb, GII.Pe, and GII.Pg associated with multiple capsid types were identified in this study, suggesting the circulation of several recombinant strains. For these strains, SimPlot analysis on ORF1/ORF2 contigs confirmed 


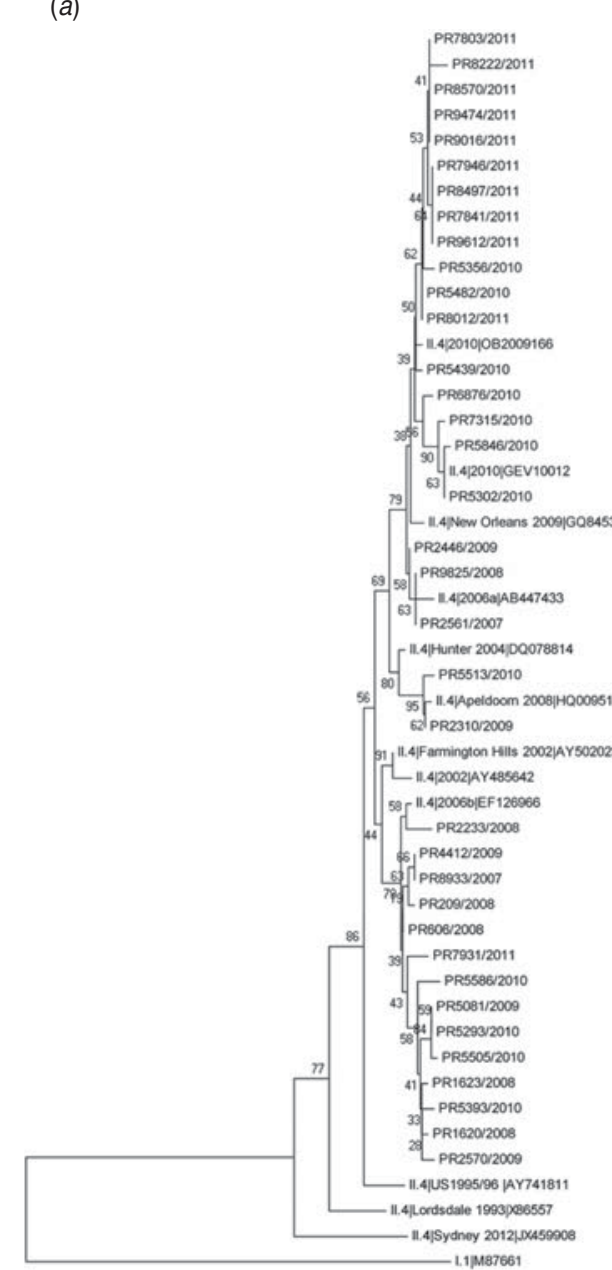

$\longmapsto$

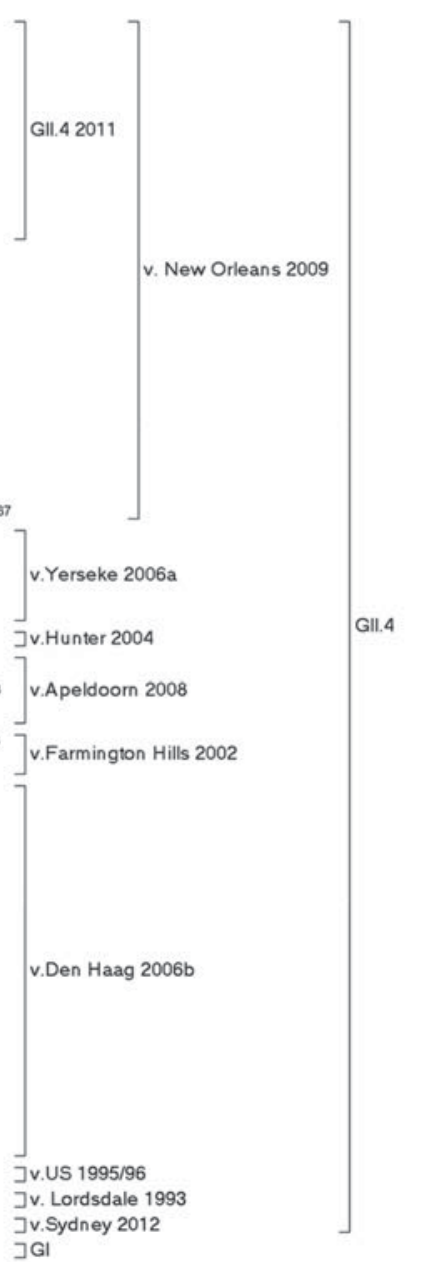

JGI
III.4

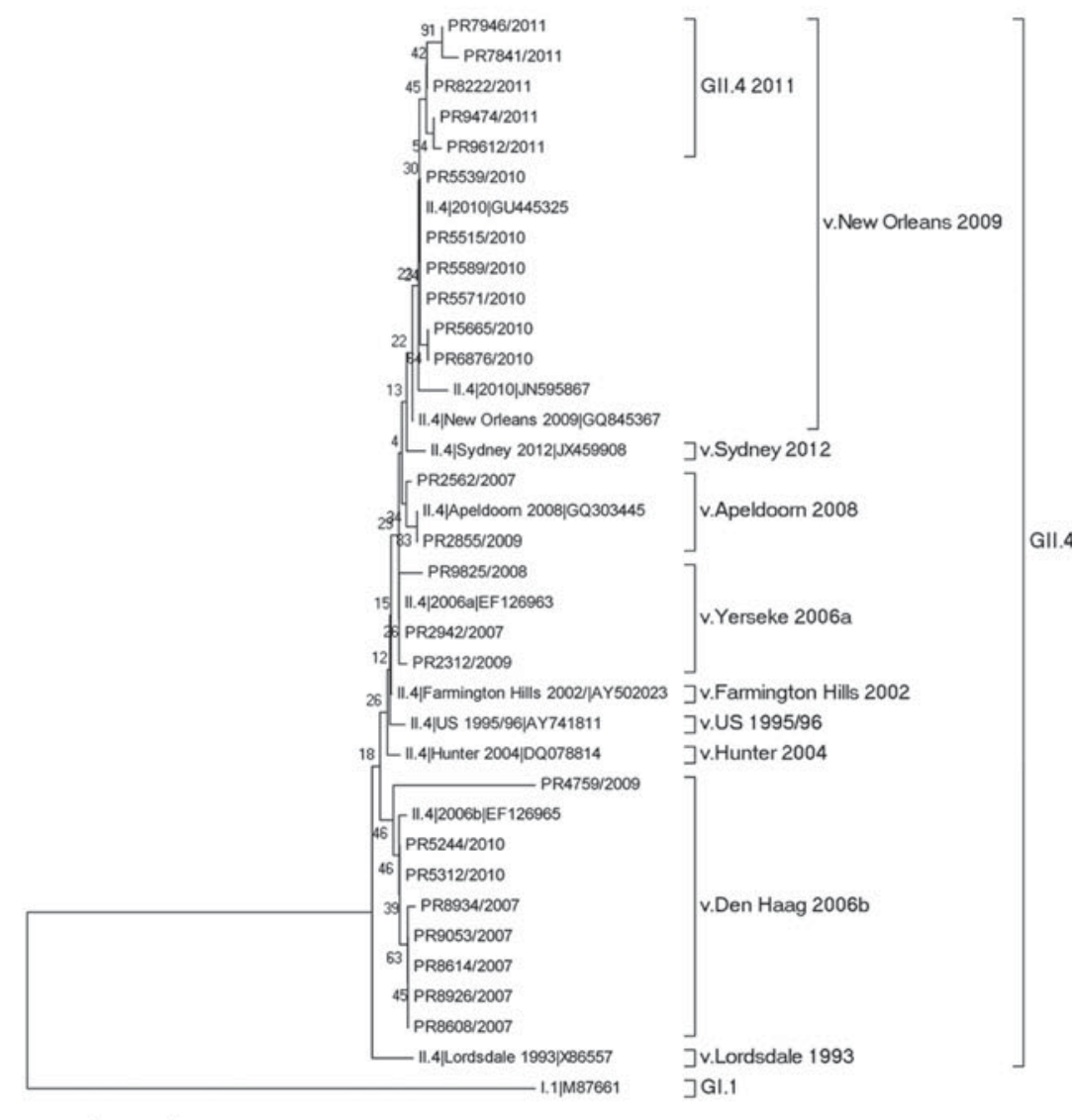

0.05

Fig. 4. (a) Neighbour-joining phylogenetic trees based on partial nucleotide sequences of norovirus polymerase gene ( $284 \mathrm{bp}$, corresponding to nucleotides $4319-4602$ referring to Lordsdale strain with GenBank accession number X86557) and (b) on partial nucleotide sequences of capsid C region (230 bp, corresponding to nucleotides 5102-5332 referring to Lordsdale strain with GenBank accession number X86557). The reference sequences were retrieved from the NoroNet database. Bootstrap values estimated with 1000 pseudo-replicate datasets, are indicated at each node. 
the recombinant nature, with the breakpoint being located in the ORF1/ORF2 junction region (data not shown). NoV recombination has been described frequently, and the crossover points are usually located at highly conserved sequence stretches, such as the ORF1/ORF2 junction region [6]. In recent years, the recombinant NoV strains GII.Pb/Hilversum have been found to be common agents of gastroenteritis in Europe, chiefly in children. GII.Pb/Hilversum NoV strains share a common polymerase gene and display a variety of capsid genes, such as GII.1 (Hawaii), GII.2 (Snow Mountain), GII.3 (Toronto) and GII.4 (Lordsdale) [16]. Interestingly, in the present study we observed 2010 NoV strains with either RdRp genotypes GII.Pe or GII.Pg apparently replacing NoV GII.Pb, which was common before 2010, suggesting a temporal shift. Many novel RdRp/capsid genotypes (GII.P7_GII.6, GII.P16_GII.3, GII.P16_GII.13, GII. Pe_GII.2, GII.Pe_GII.4 2007) were identified for the first time in sporadic cases of acute gastroenteritis in Italy, confirming that multi-target (ORF1- and ORF2-based) analysis is required in order to characterize properly NoV strains.

In conclusion, during a 5-year surveillance period, GII.4 were the predominant NoV strains in Northern Italy. Moreover, a candidate novel GII.4 variant was identified and a variety of NoV recombinants were detected for the first time in the country.

Since the epidemiology of NoV changes rapidly, enhanced monitoring of circulating NoVs will allow us to understand the current state of NoV infections in the population and support appropriate future public health intervention strategies against this important cause of sporadic childhood gastroenteritis.

\section{ACKNOWLEDGEMENTS}

This study was partly supported by the grants national project PRIN 2008 (20084F4P7X_004) granted by the Italian Ministry of Education, University and Research.

\section{DECLARATION OF INTEREST}

None.

\section{REFERENCES}

1. Koopmans M. Progress in understanding norovirus epidemiology. Current Opinion in Infectious Diseases 2008; 21: $544-552$.
2. Zeng M, et al. Clinical and molecular epidemiology of norovirus infection in childhood diarrhea in China. Journal of Medical Virology 2012; 84: 145-151.

3. Ferreira MS, et al. Assessment of gastroenteric viruses frequency in a children's day care center in Rio De Janeiro, Brazil: a fifteen year study (1994-2008). PLoS One 2012; 7: e33754.

4. Patel MM, et al. Systematic literature review of role of noroviruses in sporadic gastroenteritis. Emerging Infectious Diseases 2008; 14: 1224-1231.

5. Green KY. Caliciviridae: the noroviruses. In: Knipe DM, Howley PM, eds. Fields Virology, 5th edn. Philadelphia: Wolters Kluwer Health/Lippincott Williams and Wilkins, 2007, pp. 949-979.

6. Bull RA, et al. Norovirus recombination in ORF1/ ORF2 overlap. Emerging Infectious Diseases 2005; 11: 1079-1085.

7. Ando T, et al. Detection and differentiation of antigenically distinct small round structured viruses (Norwalk-like viruses) by reverse transcription-PCR and Southern hybridization. Journal of Clinical Microbiology 1995; 33: 64-71.

8. Vinjé J, Koopmans M. Molecular detection and epidemiology of small round structured viruses in outbreaks of gastroenteritis in the Netherlands. Journal of Infectious Diseases 1996; 174: 610-615.

9. Medici MC, et al. Broadly reactive nested reverse trascription-PCR using an internal standard control for the detection of noroviruses in stool sample. Journal of Clinical Microbiology 2005; 43: 3773-3778.

10. Yuen LK, et al. Heminested multiplex reverse transcription-PCR for detection and differentiation of Norwalk-like virus genogroups 1 and 2 in fecal samples. Journal of Clinical Microbiology 2001; 39: 2690-2694.

11. Vinjé J, Hamidjaja RA, Sobsey MD. Development and application of a capsid VP1 (region D) based reverse transcription PCR assay for genotyping of genogroup I and II noroviruses. Journal of Virological Methods 2004; 116: 109-117.

12. Kageyama T, et al. Coexistence of multiple genotypes, including newly identified genotypes, in outbreaks of gastroenteritis due to Norovirus in Japan. Journal of Clinical Microbiology 2004; 42: 2988-2995.

13. Noel JS, et al. Correlation of patient immune responses with genetically characterized small round structured viruses involved in outbreaks of nonbacterial acute gastroenteritis in the United States, 1990 to 1995. Journal of Medical Virology 1997; 53: 372-383.

14. Kojima S, et al. Genogroup-specific PCR primers for detection of Norwalk-like viruses. Journal of Virological Methods 2002; 100: 107-114.

15. Kroneman A, et al. Proposal for a unified norovirus nomenclature and genotyping. Archives of Virology 2013; 158: 2059-2068.

16. Hoa Tran TN, et al. Molecular epidemiology of noroviruses associated with acute sporadic gastroenteritis in children: global distribution of genogroups, genotypes and GII.4 variants. Journal of Clinical Virology 2013; 3: 185-193. 
17. Siebenga JJ, et al. Norovirus illness is a global problem: emergence and spread of norovirus GII.4 variants, 2001-2007. Journal of Infectious Diseases 2009; 200: 802-812.

18. Reuter G, Pankovics P, Szucs G. Genetic drift of norovirus genotype GII-4 in seven consecutive epidemic seasons in Hungary. Journal of Clinical Virology 2007; 42: $135-140$.

19. Bull RA, et al. Emergence of a new norovirus genotype II.4 variant associated with global outbreaks of gastroenteritis. Journal of Clinical Microbiology 2006; 44: 327-333.

20. Bull RA, et al. Rapid evolution of pandemic noroviruses of the GII.4 lineage. PLoS Pathogens 2010; 6: e1000831.

21. van Beek $\mathbf{J}$, et al. Indications for worldwide increased norovirus activity associated with emergence of a new variant of genotype II.4, late 2012. Eurosurveillance 2013; 18: 8-9.

22. Medici MC, et al. Molecular epidemiology of norovirus infections in sporadic cases of viral gastroenteritis among children in Northern Italy. Journal of Medical Virology 2006; 78: 1486-1492.

23. Melnick JL, et al. Lyophilized combination pools of enterovirus equine antisera: Preparation and test procedure for identification of field strains of enterovirus. Bulletin of the World Health Organization 1979; 48: 263-268.

24. Melnick JL, Wimberly IL. Lyophilized combination pools of enterovirus equine sera. New LBM pools prepared from reserves of antisera stored frozen for two decades. Bulletin of the World Health Organization 1985; 63: 543-550.

25. Medici MC, et al. Epidemiological aspects of human rotavirus infection in children hospitalized with acute gastroenteritis in an area of Northern Italy. Acta biomedica de l'Ateneo parmense 2004; 2: 100-106.

26. Arcangeletti MC, et al. Electron microscopy as a reliable tool for rapid and conventional detection of enteric viral agents: a five-year experience report. Acta bio-medica de l'Ateneo parmense 2005; 76: 165-170.

27. Kroneman A, et al. An automated genotyping tool for enteroviruses and noroviruses. Journal of Clinical Virology 2011; 51: 121-125.

28. Tamura K, et al. MEGA5: molecular evolutionary genetics analysis using maximum likelihood, evolutionary distance, and maximum parsimony methods. Molecular Biology and Evolution 2011; 28: 2731-2739.

29. Bereciartu A, Bok K, Gómez J. Identification of viral agents causing gastroenteritis among children in Buenos Aires, Argentina. Journal of Clinical Virology 2002; 25: 197-203.
30. Dey SK, et al. Molecular and epidemiological trend of norovirus associated gastroenteritis in Dhaka City, Bangladesh. Journal of Clinical Virology 2007; 40: 218-223.

31. Guo L, et al. Genetic analysis of norovirus in children affected with acute gastroenteritis in Beijing, 2004-2007. Journal of Clinical Virology 2009; 44: 94-98.

32. O'Ryan ML, et al. Prospective characterization of norovirus compared with rotavirus acute diarrhea episodes in chilean children. Pediatric Infectious Disease Journal 2010; 29: 855-859.

33. Abugalia M, et al. Clinical features and molecular epidemiology of rotavirus and norovirus infections in Libyan children. Journal of Medical Virology 2011; 83: 1849-1856.

34. Chan-It W, et al. Detection and genetic characterization of norovirus infections in children with acute gastroenteritis in Japan, 2007-2009. Clinical Laboratory 2011; 57: 213-220.

35. Kirby A, Ashton L, Hart IJ. Detection of norovirus infection in the hospital setting using vomit samples. Journal of Clinical Virology 2011; 51: 86-87.

36. Rivera FP, et al. Norovirus prevalence in 'pathogen negative' gastroenteritis in children from periurban areas in Lima, Peru. Transactions of the Royal Society of Tropical Medicine and Hygiene 2011; 105: 734 736.

37. Zeng M, et al. Clinical and molecular epidemiology of norovirus infection in childhood diarrhea in China. Journal of Medical Virology 2012; 84: 145-151.

38. Okame M, et al. Existence of multiple genotypes associated with acute gastroenteritis during 6-year survey of norovirus infection in Japan. Journal of Medical Virology 2006; 78: 1318-1324.

39. Chhabra P, et al. Epidemiological, clinical, and molecular features of norovirus infections in western India. Journal of Medical Virology 2009; 81: 922-932.

40. Junquera CG, et al. Prevalence and clinical characteristics of norovirus gastroenteritis among hospitalized children in Spain. Pediatric Infectious Disease Journal 2009; 28: 604-607.

41. Mounts AW, et al. Cold weather seasonality of gastroenteritis associated with Norwalk-like viruses. Journal of Infectious Diseases 2000; 181: 284-287.

42. Lopman B, et al. Host, weather and virological factors drive norovirus epidemiology: time-series analysis of laboratory surveillance data in England and Wales. PLoS One 2009; 4: e6671.

43. Dey SK, et al. Novel recombinant norovirus in Japan. Virus Genes 2010; 40: 362-364. 